\title{
A Biophysically-Based Model of the Optical Properties of Skin Aging
}

\author{
Jose A. Iglesias-Guitian Carlos Aliaga Adrian Jarabo Diego Gutierrez
}

Universidad de Zaragoza

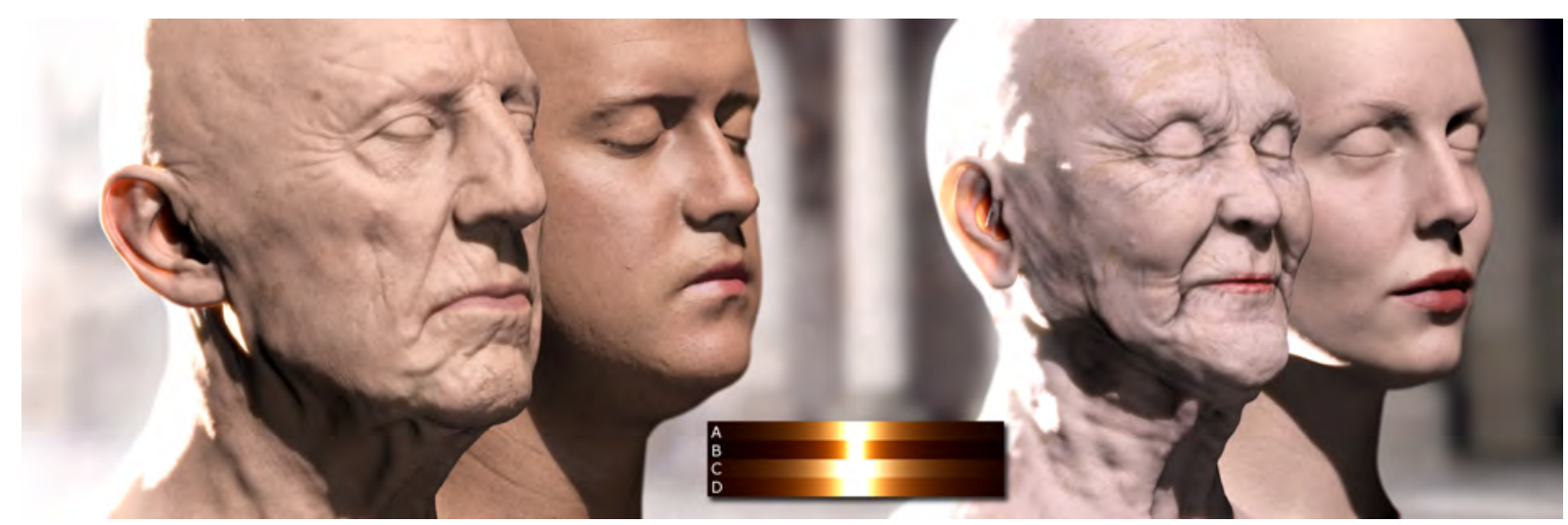

Figure 1: Our biophysically-based model allows to reproduce the changes in the optical properties of skin due to aging, which greatly affects its appearance. Structural changes and varying chromophore concentrations yield changes in the scattering and absorption coefficients, producing paler and slightly more translucent skin. The image shows examples for 30-and 80-year-olds, with mediterranean skin (type III) for the male, and caucasian (type I) for the female. The small inset shows the very different scattering profiles for each one: $A$ and $B$ correspond to the old and young males respectively, while $C$ and $D$ correspond to the old and young females. All the rendered images in the paper are best viewed in the digital version.

\begin{abstract}
This paper presents a time-varying, multi-layered biophysically-based model of the optical properties of human skin, suitable for simulating appearance changes due to aging. We have identified the key aspects that cause such changes, both in terms of the structure of skin and its chromophore concentrations, and rely on the extensive medical and optical tissue literature for accurate data. Our model can be expressed in terms of biophysical parameters, optical parameters commonly used in graphics and rendering (such as spectral absorption and scattering coefficients), or more intuitively with higher-level parameters such as age, gender, skin care or skin type. It can be used with any rendering algorithm that uses diffusion profiles, and it allows to automatically simulate different types of skin at different stages of aging, avoiding the need for artistic input or costly capture processes.
\end{abstract}

\section{Introduction}

Human skin changes significantly with age. It becomes thinner and more dry, while the concentration of chromophores (the main skin pigments) diminishes and becomes more irregular [AP81, FMM10a, $\mathrm{NBH}^{*}$ 98]. All these changes affect its optical properties, which in turn yield visible changes in its color, translucency, apparent roughness, and consequently its final appearance. Current techniques to simulate aged skin either rely on artistic skills, or require capture sessions with subjects

\footnotetext{
(c) 2015 The Author(s)

Computer Graphics Forum (c) 2015 The Eurographics Association and John

Wiley \& Sons Ltd. Published by John Wiley \& Sons Ltd.
} 
of the appropriate age. However, simulating the changes in appearance for one single subject remains an open problem.

We present a comprehensive biophysically-based model of human skin suitable for simulating skin aging. We rely on existing in-vivo and ex-vivo measurements from the extensive medical and tissue optics literature. Our multi-layered model identifies and takes into account the key structural changes (such as the slimming of the dermis and epidermis, the flattening of the dermoepidermal junction or the deterioration of the dermal collagen fibers), as well as changes in the concentration of its main chromophores (such as melanin, hemoglobin or water). This naturally leads to changes in the scattering and absorption properties of skin, which translate into considerable appearance variations.

In particular, we reproduce the changes in the base color, translucency and skin surface reflectance. Current models (e.g. [KB04, BK10, DJ05, DJ06]) are either not suitable to simulate aging, or assume many simplifications that ignore some key aspects of aging. In our work, we focus on optical properties, and do not simulate coarser structural changes like the production of wrinkles. Our model can be formulated in terms commonly used in graphics, such as absorption or scattering spectral coefficients, or even intuitive descriptors like age, gender, skin care or skin type, and can be easily integrated into existing rendering systems capable of handling diffusion profiles.

\section{Previous Work}

There is a vast number of studies about human skin in the medical and tissue optics literature [Tuc07, $\mathrm{NBH}^{*} 98, \mathrm{FMM}^{*} 10 \mathrm{~b}$, INN07]. We refer the reader to these excellent sources, and focus here on works more closely related to computer graphics.

Simulating skin. Some of the first rendered skin images used measured isotropic BRDFs [MWL ${ }^{*}$ 99]; multiple subsurface scattering (SSS) was later added by Stam [Sta01]. One of biggest breakthroughs came with the introduction in computer graphics of the dipole diffusion approximation [JMLH01], a model that was quickly made more efficient in subsequent work [JB02]. Donner and Jensen [DJ05] presented a BSSRDF model capable of dealing with layered materials, while D'Eon et al. [dLE07] approximated the diffusion profiles of translucent layers by a sum of Gaussians. The approach was later transferred to screen-space by Jimenez et al. [JSG09, JZJ*15], including transmittance [JWSG10]. Recently, the quantized diffusion model [dI11] and the subsequent photon beam diffusion [HCJ13] provide more accurate and still efficient solutions to the general problem of subsurface scattering, including skin. All these works focus on the simulation of light transport, while we focus on the optical properties of a biophysically accurate model.

Other works focus on acquiring the reflectance properties of human skin [MWL*99, AMD01]. In the case of human faces, Debevec and his team have developed increasingly sophisticated methods from the original light stage [DHT* 00$]$, where multiple images of a given subject are acquired under varying illumination conditions. These are then used to reconstruct the reflectance functions of the acquired model, allowing them to produce impressive results (e.g. [MHP*07, GHP*08, GFT*11]). Weyrich et al. [WMP* 06] developed a model based on measurements of 149 subjects, whose parameters can then be edited. Recently, Graham et al [GTB*13] synthesize high-resolution facial surface microstructures based on captured patches of microgeometry.

Tsumura et al. [THM99, TOS*03] developed an image-based technique for separating spatial distributions of melanin and hemoglobin in human skin by independent-component analysis of a skin color image. Donner and Jensen [DJ06] introduced a two-layer skin model whose parameters controlled the amount of oil, melanin and hemoglobin in the skin. Later, a multi-layered model was presented, with spatially-varying absorption and scattering parameters [ $\mathrm{DWd}^{*} 08$ ]. Jimenez et al. [JSB $\left.{ }^{*} 10\right]$ modeled changes in appearance due to varying concentrations of melanin and hemoglobin, caused by different emotional or physical states. Possibly the most detailed skin model in computer graphics nowadays is the work by Krishnaswamy and Baranoski [KB04,BK10]. However, many key aspects that change with age and affect its optical properties are not taken into account.

Aging in humans. Not many works in graphics deal with aging in humans. Two notable exceptions are the works of Golovinskiy et al. [GMP*06], who presented a statistical model that allows to synthesize wrinkles and other fine geometric features due to aging, and Boissieux et al. [BKTK00], who proposed image and model based methods to simulate wrinkles. Their works are therefore complementary to ours.

\section{Anatomy and Optical Properties of Skin}

We provide a brief overview of the anatomy and the optical properties influencing light transport in human skin, as well as the main effects of aging; in Section 4 we will present our biophysically-based model for aging of human skin. Skin is composed of three main layers: epidermis, dermis and hypodermis, with additional sub-layers (see Figure 2). The optical properties of these layers are described by the reflection off its surface, as well as the absorption and scattering events produced in the randomly inhomogeneous distribution of blood, chromophores and other components. Practical measurements of these events are expressed in the form of an absorption coefficient $\mu_{a}$ and a reduced scattering coefficient $\mu_{s}^{\prime}$. Absorption and scattering coefficients represent how much light is absorbed or scattered by the medium per incremental path length traveled. In the case of reduced scattering coefficients, we assume a diffuse light measurement, having then an isotropic phase function $(g=0)$. Spectral reflectance curves are commonly used to describe skin appearance.

The epidermis is the outermost layer of the skin, and is divided in two sub-layers: The stratum corneum, composed of many flattened and packed dead cells, is relatively high on lipids and low on water content. Light reflection off this layer is determined by surface topography and sebum production [YMOI12,PSD79]. The second layer is the living epidermis, 


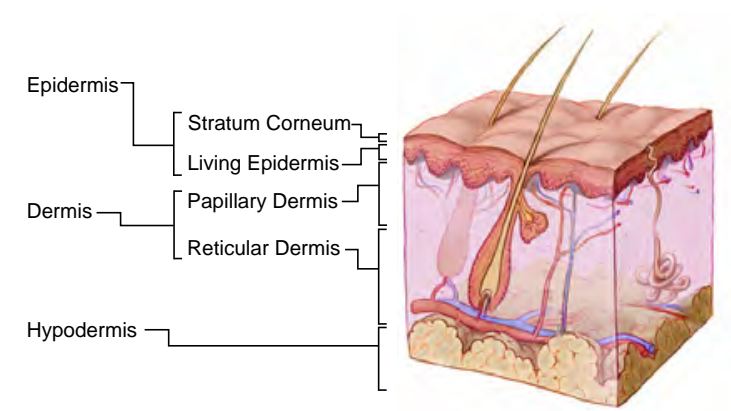

Figure 2: Skin anatomy showing the main layers present in skin (credits: Don Bliss, National Institutes of Health, US).

which primarily contains living cells like the melanosomes, responsible for the storage, transport and synthesis of melanin. This is the main light absorber of the epidermis (together with, to a much lesser extent, dietary carotenoids), and therefore its main colouring pigment. Melanosomes and melanin exhibit forward and isotropic scattering respectively [BGT11].

The dermis is generally described as a dense irregular connective tissue. It has an inhomogeneous distribution of blood vessels in its two-main sub-layers: The papillary dermis, which is a thin layer of connective tissue that interdigitates with ridges of the epidermis and contains many capillary loops and elastin fibers; and the reticular dermis, containing thick bundles of collagen, more elastin fibers, arteries and veins. The main absorbers in the dermis are blood hemoglobin, in both oxygen saturated (oxy) and desaturated forms (deoxy), carotene, bilirubin and water. The scattering properties of the dermal layers are defined by collagen fibril distributions, packed in bundles. Quantitative studies of dermal collagen provide morphometrical measurements showing histograms of fiber distributions per dermal area [BBF* 91$]$. Given its larger thickness, scattering in skin is dominated by the reticular dermis. The interface between the epidermis and the dermis is called the dermoepidermal junction.

Last, the hypodermis is adipose tissue which may be up to a few centimeters thick or completely absent, depending on its location. Absorption in the hypodermis is mainly determined by hemoglobin, lipids and water. The main scatterers in this layer are spherical droplets of lipids.

\subsection{Skin aging}

The aging process in human skin causes variations in both its structure and its main constituents. In particular:

- The epidermal and dermal layers become slimmer, with the exception of the stratum corneum. Skin becomes drier in general (see Subsection 4.1).

- The dermoepidermal junction becomes flatter (4.1).

- The roughness of the epidermis increases (4.2).

- The production of sebum in the epidermis decreases (4.2).
- The concentration of principal chromophores decrease (e.g. melanin or hemoglobin) (4.3).

- Dermal collagen fibers deteriorate (4.4).

All this leads to visible changes in the optical properties of skin, thus changing its appearance (see Figure 3). Previous skin models [DJ06, KB04] simplify the structure of the skin, allowing to simulate only a subset of the changes enumerated above. Accurately simulating all these changes requires a finer structural and biophysical granularity. We thus first develop a complete model including accurate descriptions of the hypodermis, water concentration, the dermoepidermal junction, the production of sebum in the epidermis or the distribution of collagen fibers.

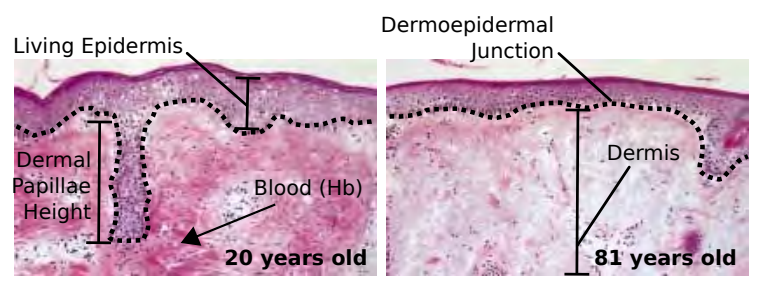

Figure 3: Skin close-ups (images augmented $\times 10$ ) for healthy subjects, ages 20 (left) and 81 (right). Epidermal thinning, flattening of the epidermal junction and the decrease of hemoglobin are clearly visible (credits: image adapted from [CYL $\left.{ }^{*} 02\right]$ ).

\section{Our Skin Aging Model}

Our model can take as input specific values from accurate measured data (i.e. tissue optics studies), or roughly approximated based on the subject's age, gender, skin type, body location and even life habits (for instance modeling the effect of excessive exposure to UV rays). While obvious differences exist in skin according to gender or skin types, the aging process is rather similar in most cases [FL86]. Therefore, unless explicitly mentioned, our aging model can be applied to both genders and all skin types.

\subsection{Layered structure}

Aging selectively affects the structural organization of skin. Capturing variations in its optical properties and therefore its color and translucency, requires a model that includes a fine detailed description of its layered structure. Our model is made up of the five main representative layers introduced in Section 3: stratum corneum (SC), living epidermis (LE), papillary dermis (PD), reticular dermis (RD) and hypodermis (HD). While the living epidermis, the papillary and reticular dermis are usually included in existing skin models, the stratum corneum and the hypodermis are commonly ignored or highly simplified. However, the former is the outmost layer, and therefore has a large impact in the final appearance, which also depends on its location and exposure to external agents (see Figure 14). The latter has been at best considered as a purely reflectance layer [KB04] or merged with other internal tissues [DJ05]; we instead include it as a fully participating medium, which leads to significant changes in 

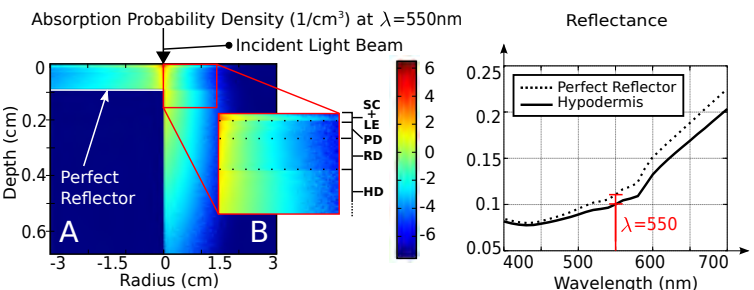

Figure 4: The influence of the hypodermis. Left: Absorption probability density for $\lambda=550$ mm a skin sample. In $A$ the hypodermis is considered to be a perfect reflector [KB04]; in B we model it as a participating layer $(d=5.9 \mathrm{~mm}, \eta=1.44)$, with absorption (Subsection 4.3) and scattering events (Subsection 4.4). The perfect reflector model in A increases absorption in the dermis and epidermis as a consequence of light being reflected off the hypodermis. Right: Spectral reflectance for both hypodermis models:accurately modeling the hypodermis reduces the amount of total outgoing energy, specially at longer wavelenghts.

the appearance of the skin, as shown in Figure 4. Our model considers each layer as an optically thick and semi-infinite (lateral extent) medium, described by its thickness $d$, index of refraction $\eta$, as well as absorption and reduced scattering coefficients $\mu_{a}$ and $\mu_{s}^{\prime}$ (see Subsections 4.3 and 4.4 respectively).

As skin ages, its rate of renewal slows down and the thickness of its layers is non-uniformly reduced, ranging from a $6.4 \%$ reduction in the living epidermis to up to $50 \%$ in the hypodermis. This large reduction in the hypodermis emphasizes the importance of this layer in a skin aging model. We model thickness atrophy by linearly reducing each layer using the values summarized in Table 1.

\begin{tabular}{ccccc}
\hline $\begin{array}{c}\text { Skin } \\
\text { layer }\end{array}$ & $\eta$ & $d(\mathrm{~mm})$ & $\begin{array}{l}\text { Structure } \\
\text { Decrease (p.d.) }\end{array}$ & Reference \\
\hline $\mathrm{SC}$ & 1.53 & 0.02 & (n.a.) & [LATO*97] \\
$\mathrm{LE}$ & 1.34 & 0.08 & $6.4 \%$ & [WM05] \\
$\mathrm{PD}$ & 1.395 & 0.18 & $6 \%-16 \%$ & [WM05] \\
$\mathrm{RD}$ & 1.39 & 1.82 & $6 \%-16 \%$ & [WM05] \\
$\mathrm{HD}$ & 1.44 & 5.9 & $30 \%-50 \%$ & [BGP66] \\
\hline
\end{tabular}

Table 1: Typical thickness of the skin layers considered in our model (base values for $t=30$ ) and their range of variation per decade (p.d.). These values give a mean plausible range corresponding to average skin [MM02].

Additionally, the dermoepidermal junction becomes progressively flatter with age (see Figure 3). We model its interdigitated nature, as proposed by Meglinsky [MM02], using a periodic surface vertically centered at the mean depth of the layer as:

$$
z_{(x, y)}=\bar{z}+A_{x} \sin \left(\omega_{x} x+\phi_{x}\right) \cdot A_{y} \sin \left(\omega_{y} y+\phi_{y}\right)
$$

where $x, y$ are coordinates lying on the plane and $z$ is depth, $\phi_{x, y}$ and $\omega_{x, y}$ are the phase offsets and frequency modeling the surface roughness, and $A_{x, y}$ is the peak amplitude of the rete ridge surface, which decreases linearly with age. The reported measurements by Giangreco et al. [GGF* ${ }^{*} 10$ ] range from $100 \mu \mathrm{m}$ to almost $0 \mu m$, for ages 20 through 80 .

\subsection{Surface reflection}

On average, about $5 \%$ of the incident light is reflected off the surface of the stratum corneum, although the actual percentage varies with hydration and sebum production. Its effect on the appearance of skin depends mainly on its roughness and oiliness [PSD79]. Tissue optics studies commonly relate roughness variations related with aging with parameterizations of a Beckmann distribution function [YMOI12]. We model the skin's outmost surface using the Torrance-Sparrow microfacet BRDF model [TS67], using the average angle $\alpha_{s r}$ of the microfacets as the Beckmann's distribution roughness. We model $\alpha_{s r}=\arctan \left(R_{z} / l_{e}\right)$, with $R_{z}$ the average peak-to-valley height from a set of measurments, and $l_{e}=960 \mu \mathrm{m}$ the skin profile length of each measurment [EHM03]. Values of $R_{z}$ increase with age, and the values used in our model are shown in Figure 5 (left).
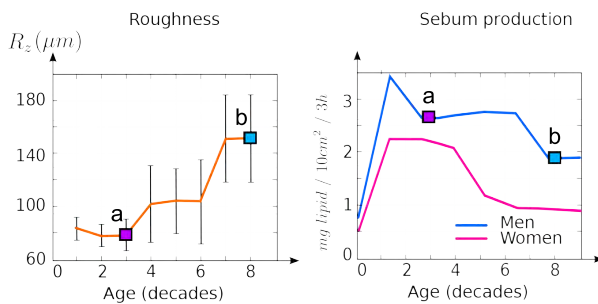

Figure 5: Left: Evolution of surface roughness $R_{z}$. Measured mean values vary from $83 \mu \mathrm{m}$ in young individuals up to $151 \mu \mathrm{m}$ in the elderly [EHM03]; Right: Evolution of sebum production, measured in mg of lipids produced in $10 \mathrm{~cm}^{2}$ after $3 \mathrm{~h}$. Surface roughness increases, while sebum production decreases in the elderly, being always greater in men than women [PSD79].

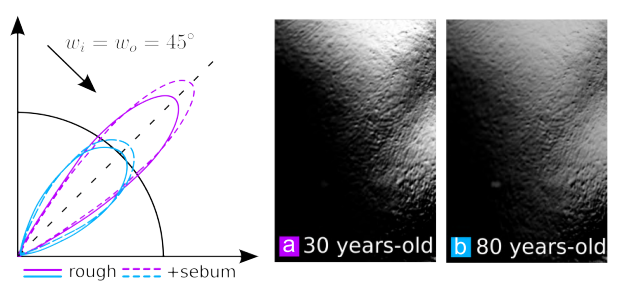

Figure 6: Effect of roughness variation with age on the specular reflection. Left: The roughness of the Beckmann distribution increases with age as a combination of both the sebum production and the skin surface roughness variations. Center: Reflectance in young skin (30 years old). Right: Reflectance in aged skin ( 80 years). In both cases, both the change of roughness and sebum is modeled (Figure 5 shows the values of a and $b$ used).

Moreover, the production of sebum creates a thin film that smoothens the skin roughness. As humans age, sebaceous 
glands become less active: the thickness $s$ of the sebum film can vary from $3 \mu \mathrm{m}$ in young skin to $1.69 \mu \mathrm{m}$ in the elderly [PSD79]. To simulate this, we assume that the average slope of the microfacets is reduced due to the sebum layer, so that $\alpha_{s r}^{\prime}=\psi_{s} \alpha_{s r}$, where $\psi_{s} \in[0 . .1]$ is a modulation factor dependent on the average sebum volume filling the microfacets (see supplementary material for details). We make an assumption about the pyramidal shape of the microfacets, but we only use that to derive the statistical roughness reduction, not the real per-facet slope variation. Figure 6 compares the specularity of skin with age, both with and without including sebum in the skin.

\subsection{Absorption}

We define the absorption coefficient $\mu_{a}$ of a skin layer as the sum of contributions for all absorbing chromophores present in that layer, which is the common way to define it in multi-layered tissue optics [Jac13]:

$$
\mu_{a}=\ln (10) \sum_{i} c_{i} \varepsilon_{i}
$$

where $\varepsilon_{i}$ is the chromophore extinction coefficient ${ }^{\dagger}$ and $c_{i}$ its concentration. Our model includes a wide range of chromophores identified in tissue optics literature as dominant light absorbers in human skin [Jac13]. Similarly to previous biophysical models for computer graphics [KB04], we include melanin (eumelanin and pheomelanin), haemoglobin $(\mathrm{Hb})$ found in blood (oxygen-saturated $\mathrm{HbO}_{2}$ and desaturated $\mathrm{Hb}$ ), as well as bilirubin and carotene. In addition, and different from previous models, we include water as the seventh chromophore, using spectral absorption measurements given by Pope and Fry [PF97]. Although water is not a highly-absorbent component itself, it has a great influence in appearance, since it defines dryness and dilutes the concentration of other chromophores (see Figure 8). Water concentrations decrease with age, causing a progressive thinning of the layers (Subsection 4.1).

The concentration of chromophores is specified for each layer in anatomical volume fractions of tissue occupied by their principal container (melanosomes in the case of melanin $\vartheta_{m}$ or whole blood in the case of hemoglobin $\vartheta_{H b}$ ). Water is expressed directly as the volume fraction of water content $f_{\mathrm{H}_{2} \mathrm{O}}$ while carotene $c_{c a r}$ and bilirubin $c_{b i l}$ are concentrations expressed in $g / L$. Table 2 shows per-layer chromophore concentrations for an adult skin sample. Last, we also estimate a baseline skin absorption, modeling depigmentation due to small-scale tissues. Our baseline is approximated from data measured by Saidi [Sai92].

The final absorption values are determined by applying Equation 2 to each of the five layers considered in our model. Figure 7 (left) shows the absorption spectral curves for all the chromophores in our model, including our skin baseline absorption. Figure 7 (right) shows an example of the spectral absorption

$\dagger$ The term $\ln (10)$ stands in Equation 2 because first spectrometers recorded $\varepsilon$ in base 10 [Jac13].

(C) 2015 The Author(s)

Computer Graphics Forum (C) 2015 The Eurographics Association and John Wiley \& Sons Ltd.

\begin{tabular}{cccccc}
\hline $\begin{array}{c}\text { Skin } \\
\text { layer }\end{array}$ & $\vartheta_{m}$ & $\vartheta_{H b}$ & $c_{\text {bil }}$ & $c_{c a r}$ & $f_{H_{2} O}$ \\
\hline SC & - & - & - & $2.1 \mathrm{e}-4$ & 0.05 \\
LE & {$[0-35] \%$} & - & - & $2.1 \mathrm{e}-4$ & 0.2 \\
PD & - & {$[0.1-20] \%$} & {$[0.05-3]$} & $7.0 \mathrm{e}-5$ & 0.5 \\
RD & - & {$[0.1-20] \%$} & {$[0.05-3]$} & $7.0 \mathrm{e}-5$ & 0.7 \\
HD & - & $5 \%$ & - & - & 0.7
\end{tabular}

Table 2: Chromophore concentrations for a typical adult. Values inside brackets indicate plausible range values [MM02].
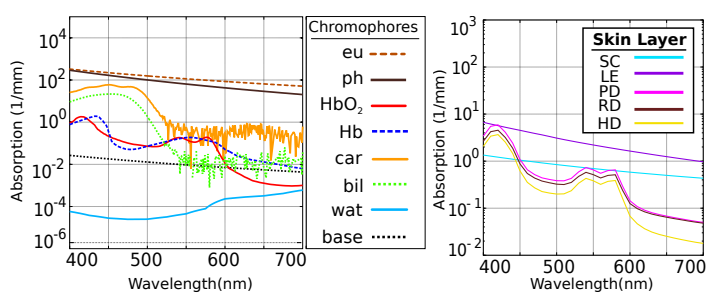

Figure 7: Left: Spectral absorption curves for the seven chromophores, plus the baseline absorption used in our model. We refer to the supplementary material for equations used to model absorption coefficients for each chromophore. Right: A representative example of the spectral absorption coefficients for the five layers considered in our model, for a 30 years old woman with skin type II $\vartheta_{m}=3 \%$ and $\vartheta_{H b}=1 \%$.

curves resulting from our model. Notice how the spectral signature of each chromophore (Figure 7, left) drives the absorption of the layers in which their concentration is higher (e.g. melanins in epidermal layers or hemoglobin in the dermal layers). Figure 8 shows the influence of water in skin, visibly reducing its spectral absorption in every layer, while increasing its reflectance. (a) Absorption per layer
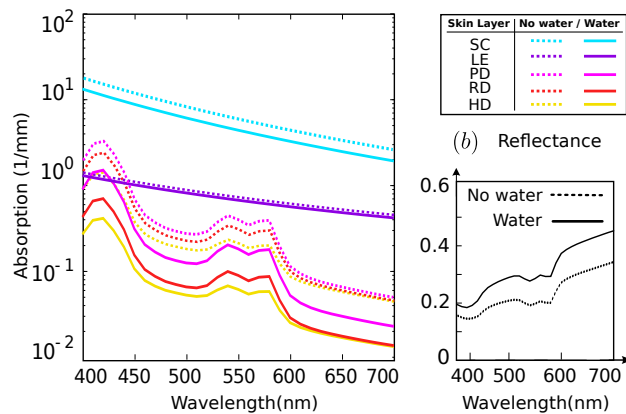

(b) Reflectance

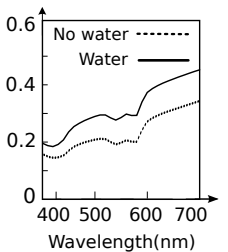

Figure 8: Influence of water on the spectral absorption of skin. Despite its low absorbance, water plays an important role in the state of skin. Notice how, by considering water concentrations, almost all layers suffer a considerable reduction in their spectral absorption, while the reflectance increases significantly. 
Decrease of melanin and hemoglobin. As mentioned before, the two main reasons for the decrease of chromophore concentration with age are the thinning of skin layers, and the loss of water in the hypodermis. Melanin and hemoglobin, however, suffer an additional decrease that strongly influences the appearance of aged skin [FMM10a, CYL*02]. In the case of melanin, its rate of decline $r_{\vartheta_{m}}$ has been measured at about $8 \%$ per decade, with no significant differences with respect to sun exposition or gender [GS79]. Given a concentration of whole blood $\vartheta_{m}$ at age $t$ in decades (Table 2) and a time interval $\Delta t$, we obtain the new concentration $v_{m}^{\prime}$ at $t+\Delta t$ as:

$$
\vartheta_{m}^{\prime}=\vartheta_{m}-\left(\Delta t \cdot r_{\vartheta_{m}}\right) \vartheta_{m}
$$

For hemoglobin, we need to consider both intrinsic and extrinsic factors. We thus model the new concentration $\vartheta_{H b}^{\prime}$ at time $t+\Delta t$ as:

$$
\vartheta_{H b}^{\prime}=\vartheta_{H b}-\Delta t\left(r_{\vartheta_{H b i}}+\xi_{\vartheta_{H b e}}\right) \vartheta_{H b}
$$

where $r_{\vartheta_{H b i}}=6 \%$ and $r_{\vartheta_{H b e}}=10 \%$ are the intrinsic and extrinsic decrease rates respectively for $\mathrm{Hb}$ concentrations [ $\mathrm{CYL}^{*} 02$ ], and $\vartheta_{H b}$ is the hemoglobin concentration at time $t$ (Table 2). Extrinsic factors like exposition to UV rays lead to a further decreased concentration of hemoglobin; they are modeled by an empirical value $\xi \in(0,1)$ representing the exposure of skin to aging by external agents. Figures 9 and 10 show the effect in appearance caused by melanin and hemoglobin reduction, respectively. Parameters like surface roughness and other chromophore concentrations are kept fixed to values shown in Tables 3 and 4 .

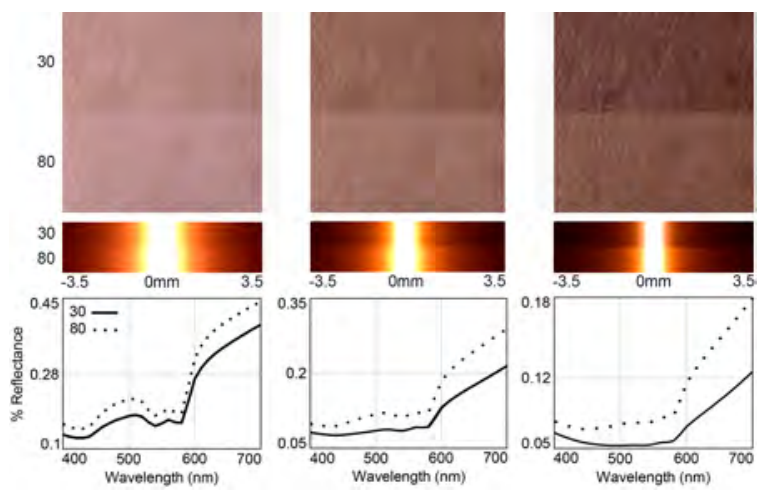

Figure 9: Influence of the decrease of melanin with age. Each column shows a patch of skin for a 30- (top) and a 80-year old (bottom), along with their corresponding scattering profiles and spectral reflectance curves. From left to right, we show light caucasian skin with $2 \%$ of melanin concentration in the epidermis, mediterranean skin (7\%), and a moderately dark skin (15\%). Melanin concentration in the 80-year-old samples is computed using Equation 3, while other model parameters are kept fixed (see Tables 3 and 4).
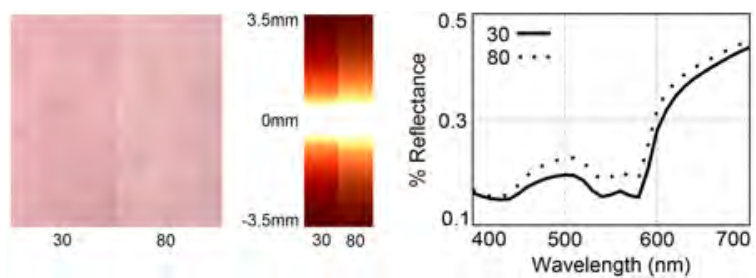

Figure 10: Influence of the decrease of hemoglobin with age. Hemoglobin concentration in the 80-year-old sample is computed using Equation 4, taking into account both intrinsic and extrinsic effects. The remaining model parameters are fixed (see Tables 3 and 4). Left: Patch of caucasian skin for a 30- (top) and a 80-year old (bottom), showing a decrease in hemoglobin concentration from $8.4 \%$ to $5.9 \%$ in the PD and from $7.4 \%$ to $5.2 \%$ in the RD. Middle: Scattering profiles. Right: Spectral reflectance curves.

\subsection{Scattering}

Contrary to absorption, there is a lack of accurate spectral scattering data for human skin across a wide range of ages. To overcome this, the spectral scattering behavior of skin can be instead characterized by wavelength-dependent mathematical power law functions fitted from existing sparse measured data [Jac13, BGT11]. This wavelength dependence can be expressed in terms of the separate contributions of Rayleigh $\left(\mu_{s}^{\prime R}\right)$ and Mie $\left(\mu_{s}^{\prime M}\right)$ scattering as:

$$
\mu_{s}^{\prime}(\lambda)=\rho \cdot \mu_{s}^{\prime R}(\lambda)+(1-\rho) \cdot \mu_{s}^{\prime M}(\lambda)
$$

where $\rho \in[0 . .1]$ indicates the proportion among Rayleigh and Mie scattering, and:

$$
\begin{aligned}
& \mu_{s}^{\prime R}(\lambda)=\mu_{s}^{\prime}\left(\lambda_{r}\right) \cdot\left(\lambda / \lambda_{r}\right)^{-4} \\
& \mu_{s}^{\prime M}(\lambda)=\mu_{s}^{\prime}\left(\lambda_{r}\right) \cdot\left(\lambda / \lambda_{r}\right)^{-\gamma}
\end{aligned}
$$

The scaling factor $\mu_{s}^{\prime}\left(\lambda_{r}\right)$ depends on the reference wavelength $\lambda_{r}=500 \mathrm{~nm}$, and the scattering factor $\gamma$ characterizes the wavelength dependence of the Mie scattering component. In the following, we describe the scattering for each layer independently.

Stratum corneum and living epidermis. The scattering of the epidermal layers is characterized by considering both the stratum corneum and the living epidermis as a whole, given the strong forward scattering characteristics of both layers and the thinness of the stratum corneum [Pen62]. Variations in melanin with age are not significant. We thus model the scattering of both layers using Equation 5 using $\rho=0.29, \mu_{s}^{\prime}\left(\lambda_{r}\right)=6.67 \mathrm{~mm}^{-} 1$ and $\gamma=0.689$ [Jac13].

Papillary and reticular dermis. For scattering in the dermal layers, previous models [DJ06] assume that the reduced scattering coefficient is halved with respect to the epidermal regions. While this assumption may be valid for stationary models, it falls short for skin aging. Light scattering in the 
dermis changes according to specific redistributions of collagen fibers, which are mainly responsible of Mie scattering. Their amount and mean diameter size differ between the papillary and reticular dermis, as shown by Branchet [BBF*91]. We thus keep Rayleigh scattering constant in our model, with $\mu_{s}^{\prime}\left(\lambda_{r}\right)=4.36 \mathrm{~mm}^{-1}$ for $\lambda_{r}=500 \mathrm{~nm}[\mathrm{Jac} 13]$, the measured fraction $\rho=0.41$ [SJNY06], and vary only the $\gamma$ parameter.

To compute $\gamma$, we assume that the distribution of diameters $d$ of collagen fibers follows a skewed normal distribution with mean $\bar{d}$ and standard deviation $\sigma$, with an skew factor $\zeta$. This allows us to model the asymmetric distributions of diameters that appear with age [ZD09, MFB ${ }^{*}$ 97]. Therefore, we obtain a probability density function $h(d)$ for a fiber diameter $d$ as:

$$
h(d)=2 \cdot \phi(d, \bar{d}, \sigma) \cdot \Phi(\zeta d, \bar{d}, \sigma),
$$

where $\phi$ is a Gaussian distribution and $\Phi$ is the cumulative distribution function of $\phi$. Note that this is a continuous function, while in morphometric analysis in tissue optics $h(d)$ is generally reported using a discretized histogram. We then model $\gamma$ for a given $d$ as an inverse linear approximation [MCD97] in the range $\left(\gamma_{\min }, \gamma_{\max }\right)$, with $\gamma_{\min }=0.2$ and $\gamma_{\max }=4$ [ZD09], with $d \in[1.15,35] \mu \mathrm{m}$.

Given a distribution $h(d)$ of fiber diameters and their corresponding $\gamma(d)$ values, we obtain $\mu_{s}^{\prime M}(\lambda)$ from Equation 7 . We finally compute $\mu_{s}^{\prime M}(\lambda)$ for both the papillary and reticular dermis as:

$$
\mu_{s}^{\prime M}(\lambda)=\frac{\sum_{d} \mu_{s}^{\prime M(\gamma(d))}(\lambda) \cdot h(d)}{\sum_{d} h(d)} .
$$

Aging and redistribution of the collagen fibers is modeled by adjusting the parameters of the diameter distribution function $h(d)$. As the mean thickness of the fibers increase with age, the model adjusts $h(d)$ around greater thickness values by proportionally adjusting $\bar{d}$. The redistribution of the fibers with increasing heterogeneity is simulated by progressively increasing $\sigma$ in the Gaussian distribution. The physical meaning of varying $\zeta$ corresponds to simulating more asymmetric distributions of fibers with time (see Figure 11). For the age range of 30-80 years, mean, variance and skew vary linearly $\left[\mathrm{BBF}^{*} 91\right]$.

Hypodermis. The main aging characteristic of the hypodermis is the loss of fat tissue. The spectral dependence of the reduced scattering coefficient for the hypodermis $\mu_{s}^{\prime h d}(\lambda)$ can be approximated from existing sparse measurements by fitting to the power law [BGKT05]:

$$
\mu_{s}^{\prime h d}(\lambda)=105.06 \lambda^{-0.68} \quad\left[\mathrm{~mm}^{-1}\right]
$$

\section{Using Our Model}

Our time-varying skin model can be plugged in any multilayered rendering framework for translucent materials (e.g. [WJZ95, DJ05, dI11]), by computing for each layer its width $d$, index of refraction $\eta$, and absorption $\mu_{a}$ and reduced scattering $\mu_{s}^{\prime}$ coefficients for a given age. This requires to set the appropriate
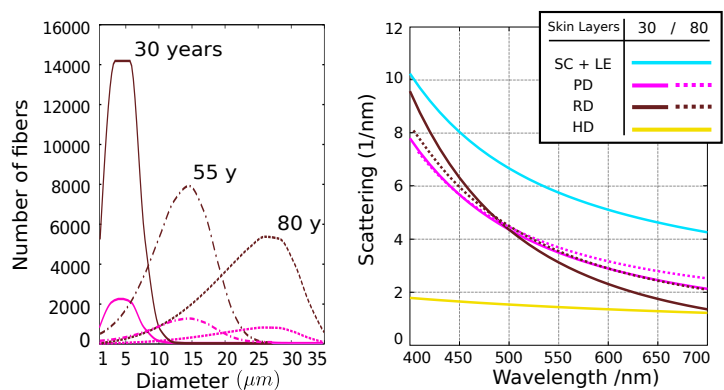

Figure 11: Left: Distributions of collagen fibers diameters obtained for papillary dermis skin samples, for ages 30, 55 and 80-years old. Right: Corresponding variations of the spectral scattering coefficient for the different skin layers.

parameters to define the type of skin (mainly melanin concentration $\vartheta_{m}$ ) and blood profusion (hemoglobin concentration $\vartheta_{H b}$ ), in addition to the gender of the subject, skin care $\xi$ and the body location of the skin patch, using the formulas provided in this paper. Note that, although in our work we fix other parameters, such as bilirubin, carotene and water concentration, these can also be tuned to simulate effects due to sickness or dehydration.

Our proposed model offers a high degree of control over its biophysical constituents, which is desirable for many fields such as medical applications. However, we also provide a database with the diffusion profiles for the six Fitzpatrick's skin types under a combination of different ages $t$ and levels of skin care $\xi$, to simplify its use (see the supplemental material). These profiles have been computed using the MCML package [AYLH*10, WJZ95], modified to include non-planar layer interfaces (Equation 1). We calculate such diffusion profiles for an incoming pencil beam perpendicular to the surface, for wavelengths in the 400 to $700 \mathrm{~nm}$ range, sampled every $10 \mathrm{~nm}$. Each diffusion profile is then transformed from spectral XYZ tri-stimulus values to sRGB color space for rendering. These profiles are given in tabulated form, and in the form of a sum of six Gaussians (following D'Eon et al. [dL07]), making them ready to use even in real-time rendering applications [dLE07, JSG09].

\section{Results}

We now show results using plausible data ranges reported on previous studies in the tissue optics literature (see Table 2). All the data used for each image is reported in Tables 3 and 4 . We demonstrate the versatility of our skin aging model generating results for a wide range of skin samples and types (please consult the supplementary material). All the rendering results in this paper have been obtained using Jensen and Buhler's hierarchical subsurface scattering [JB02] implemented in PBRT [PH10]. We use tabulated RGB diffusion profiles obtained from multilayered simulations from the MCML package as explained in Section 5. 

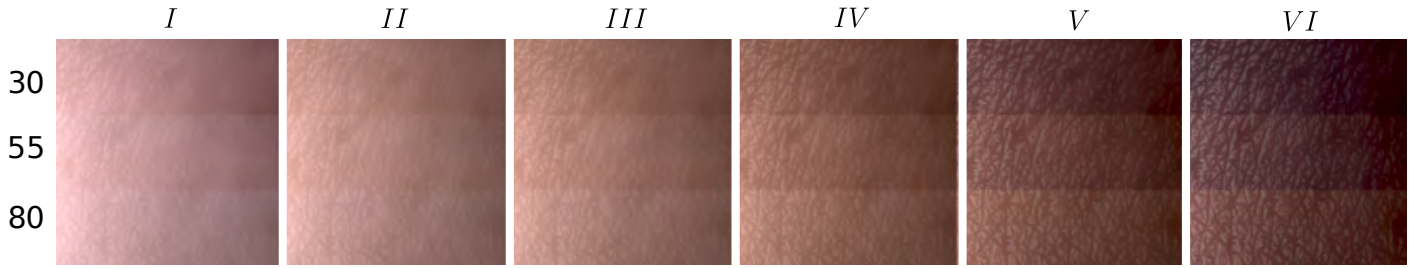

Figure 12: Results of aging a skin patch for the six types of skin according to the Fitzpatrick scale [Fit88]. The skin type is mostly defined by the concentration of melanin $\vartheta_{m}$, as: type I, $\vartheta_{m}<3 \%$; type II, $\vartheta_{m} \in[3-5) \%$; type III, $\vartheta_{m} \in[5-15) \%$; type IV, $\vartheta_{m} \in[15-25) \%$; type $V, \vartheta_{m} \in[25-35) \%$; and type VI, $\vartheta_{m} \geq 35 \%$.

Figure 12 shows how our skin model is able to reproduce appearance changes due to aging for different types of skin. We show the subtle but noticeable changes in color due to intrinsic and extrinsic age-related changes in the tissue structure and composition. Additionally, the change of shape of the specular lobes gives a more glossy appearance to young skin. Figure 1 shows two additional examples of the change in appearance of skin due to aging, for a male and female of skin types III and I respectively. Heterogeneities are modeled using melanin and hemoglobin concentration maps [DWd*08, JSB* 10 ] (see the supplementary material for details). Close-ups for a skin type II can be seen in Figure 13. Notice the increased translucency in the ears, as well as the overall loss of pigment due to the lower chromophore concentration.

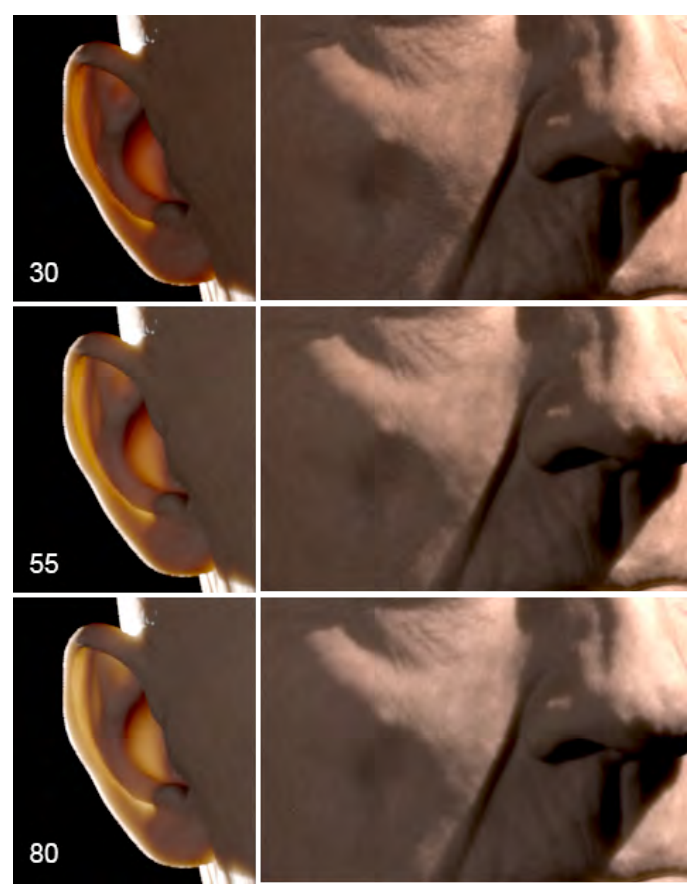

Figure 13: Differences on skin translucency and overall color between 30, 55 and 80 years old, for a subject with skin type II (note that we do not change the geometry for a more direct comparison).
Last, Figure 14 shows the appearance difference for skin of the same subject belonging to different body locations: the palm and the back of the hand. It can be seen how changes are much obvious in the back: this is due both to its different skin structure and chromophore concentrations, and to its higher sensibility to extrinsic factors: the palm suffers less impact since its outermost layers are $4-5$ times thicker.
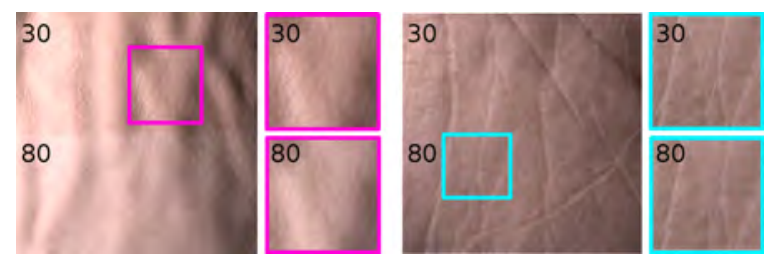

Figure 14: Aging effects for the same skin type, 30 and 80 years, but from two different body locations: back of the hand (left), and palm (right). As in other aging examples, the skin becomes less glossy and more pale. However, the palm is less sensible to extrinsic factors, due to its thick outer layers [EHT07, WE73].

\subsection{A simplified model}

The completeness and associated complexity of our model is important for certain application domains where the accuracy of the simulation plays an important role, such as medical and dermatology studies, or the cosmetic industry. Aging is the result of a complex combination of parallel processes causing changes on both the structure and composition of the skin's constitutive tissues (see Section 3); thus, a finer granularity than previous approaches is needed. However, less demanding applications, such as games, may capture the main optical effects of aging using a simplified version of our model. While the results will not be as accurate, this flexible tradeoff between complexity and accuracy increases the applicability of our model. We propose one such simplified version, which only uses three layers. For the epidermis, we consider a single layer containing the same chromophores and concentrations of the living-epidermis, but occupying the aggregated thickness of the living-epidermis and the stratum-corneum together. A similar approach is followed for the dermis, adding the thickness of both the papillary and the reticular dermis, assuming the characteristics of the latter. To further 


\begin{tabular}{cccccc}
\hline Figure & \multicolumn{5}{c}{ Input Model Parameters } \\
\hline & Age & Gender & Body Location & Skin Type & $\xi$ (External Agents) \\
\hline 1 - a & 80 & Male & Face & III & 0.5 \\
1 - b & 30 & Male & Face & III & 1 \\
1 - c & 80 & Female & Face & I & 0.5 \\
1 - d & 30 & Female & Face & I & 1 \\
9 & $30(80)$ & Female & Forearm & I, III, IV & 1 \\
10 & $30(80)$ & Female & Forearm & I & 0 \\
12 & $30(55,80)$ & Female & Forearm & I,II,III,IV,V,VI & 1 \\
13 & $30(55,80)$ & Male & Face & III & 1 \\
14-Left & $30(80)$ & Male & Hand - back & III & 1 \\
14 Right & $30(80)$ & Male & Hand - palm & III & 1 \\
15(5L) & $30(80)$ & Female & Forearm & III & 1 \\
15(3L) & $30(80)$ & Female & Forearm & III & 1 \\
\hline
\end{tabular}

Table 3: Input parameters for the images shown in the paper. Skin type is specified by the Fitzpatrick scale. Skin care $\xi$ ranges from 0 to 1. Aged samples are represented within parentesis.

simplify our model, the dermoepidermal junction is considered flat with a constant collagen fiber density. Figure 15 (left) shows a patch of mediterranean skin (type III) for ages 30 and 80, using this simplified three-layered version, and our complete model. Since tone mapping tends to equalize the images, the false color version to the right, as well as the scattering profiles in Figure 15 (right), highlight the differences between the two; we can see the differences between the two models, and how the errors in the predicted scattering are larger for the simplified aged version of skin.

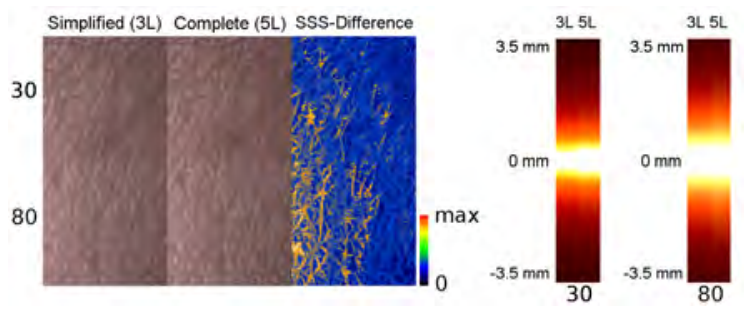

Figure 15: Left: Differences when using a simplified, threelayered version (3L) of our original model (5L). Right: Comparison of the scattering profiles pairs showing 30 and 80-year-old skin patches, using the simplified version and our complete model. Again, the differences are noticeable.

\section{Conclusions and Future Work}

We have presented a comprehensive, biophysically-based skin model capable to simulate the effects of aging, without the need for skilled artistic input or subject-specific capture sessions. It is based on a detailed multilayered structure (including the hypodermis and the dermoepidermal junction), the most dominant chromophores, and scattering profiles fitted from sparse measured data (including novel aspects such as the distribution of collagen fibers). Our model can simulate the appearance changes due to intrinsic and extrinsic skin aging, and can be expressed in terms of low-level biophysical parameters or, more intuitively, with high-level parameters such as age, gender or skin type. Our tabulated diffusion profiles allow it to be easily integrated in real-time applications such as games or medical simulations. Although our skin model requires more structural complexity than previous approaches, it can be used to obtain a diffusion profile similar to previous works, so this required complexity does not affect the rendering stage: As described in the paper, our model can be directly plugged into any existing rendering system capable of handling diffusion-based subsurface scattering without further modifications.

Our model offers a potential range of applications beyond graphics. For instance, its predictive nature can be useful in fields such as cosmetics, dermatology, or tissue optics. We are currently bounded by existing measurements and collected data. For instance, there is no existing bio-physical data about heterogeneities, which could nevertheless be added to our model using textures modulating the spatially-varying distribution of chromophores or the albedo of skin, similar to previous works (e.g. [JSB* 10, DWd*08]). An interesting avenue of future work would be modelling the time-varying nature of these heterogeneities based on a data-driven approach. We hope that our paper helps stimulate the creation of more complete datasets, although this will be a long-term effort.

Acknowledgments We thank Dr. Maria P. Matilde for our insightful discussions about skin aging, Jose I. Echevarria for his help putting together the manuscript, and Sergio Garcia, Alex Huguet and Zbynek Kysela for the head models. This research has been partially funded by the EU FP7 Program, through projects GOLEM and VERVE, as well as the Spanish Ministry of Economy and Competitiveness through project LIGHTSLICE.

\section{References}

[AMD01] Angelopoulo E., Molana R., Danillidis K Multispectral skin color modeling. In Computer Vision and Pattern Recognition, 2001. CVPR 2001. Proceedings of the 2001 IEEE Computer Society Conference on (2001), vol. 2, pp. II-635-II-642 vol.2. 2

[AP81] ANDERSON R. R., PARRISH J. A.: The optics of human skin. Journal of Investigative Dermatology 77, 1 (1981). 1

[AYLH*10] Alerstam E., Yip Lo W. C., Han T. D., Rose J., ANDERSSON-ENGELS S., LILGE L.: Next-generation acceleration and code optimization for light transport in turbid media using gpus. Biomedical optics express 1, 2 (2010). 7

[BBF*91] Branchet M., Boisnic S., Frances C., Lesty C., ROBERT L.: Morphometric analysis of dermal collagen fibers in normal human skin as a function of age. Archives of gerontology and geriatrics 13, 1 (1991). 3, 7

[BGKT05] BashKatov A., Genina E., Kochubey V., Tuchin V.: Optical properties of human skin, subcutaneous and mucous tissues in the wavelength range from 400 to $2000 \mathrm{~nm}$. Journal of Physics D: Applied Physics 38, 15 (2005). 7

[BGP66] Booth R. D., Goddard B., Paton A.: Measurement of fat thickness in man: a comparison of ultrasound, harpenden calipers and electrical conductivity. British Journal of Nutrition 20, 04 (1966). 4

[BGT11] Bashkatov A. N., Genina E. A., Tuchin V. V.: Optical properties of skin, subcutaneous, and muscle tissues: a review. Journal of Innovative Optical Health Sciences 4, 01 (2011). 3, 6 
Iglesias-Guitian et al. / A Biophysically-Based Model of the Optical Properties of Skin Aging

\begin{tabular}{|c|c|c|c|c|c|c|c|c|c|c|c|}
\hline \multirow[t]{3}{*}{ Figure } & \multicolumn{11}{|c|}{ Intermediate and Output Model Parameters } \\
\hline & \multicolumn{5}{|c|}{ Thickness (mm) } & \multicolumn{3}{|c|}{ Main Chromophores Concentrations $(\%)$} & \multicolumn{3}{|c|}{ Surface Reflection } \\
\hline & $\mathrm{SC}$ & LE & PD & $\mathrm{RD}$ & $\mathrm{HD}$ & $\vartheta_{m}(\mathrm{LE})$ & $\vartheta_{H b}(\mathrm{PD})$ & $\vartheta_{H b}(\mathrm{RD})$ & $R_{z}(\mu m)$ & $\begin{array}{l}\text { Sebum prod. } \\
\left(\frac{m g \text { lipid }}{10 \mathrm{~cm}^{2}} / 3 h\right)\end{array}$ & $\mathrm{m}$ \\
\hline $1-\mathrm{a}$ & .02 & .051 & .13 & 1.28 & 5.9 & 7 & 2.4 & 1.8 & 151 & 1.69 & 0.143 \\
\hline $1-b$ & .02 & .08 & 18 & 1.82 & 5.9 & 7 & 2.4 & 1.8 & 78 & 2.4 & 0.068 \\
\hline $1-\mathrm{c}$ & .02 & .06 & .13 & 1.27 & 5.9 & 2 & 1.2 & 0.9 & 151 & 0.85 & 0.148 \\
\hline $1-d$ & .02 & .08 & .18 & 1.82 & 5.9 & 2 & 1.2 & 0.9 & 78 & 2 & 0.069 \\
\hline 9 & $.02(c)$ & $.08(c)$ & $.18(c)$ & $1.82(c)$ & $5.9(c)$ & $2(1.2), 7(4.2), 15(9)$ & $6(c)$ & $4.5(c)$ & $78(c)$ & $2.4(c)$ & $0.068(c)$ \\
\hline 10 & $.02(c)$ & $.08(c)$ & $.18(c)$ & $1.82(c)$ & $5.9(c)$ & $1(c)$ & $8.4(5.8)$ & $7.4(5.216)$ & $78(c)$ & $2.4(c)$ & $0.068(c)$ \\
\hline 12 & .02 & $.08(.07, .06)$ & $.18(.15, .13)$ & $1.82(1.55,1.27)$ & 5.9 & $\begin{array}{c}1(0.8,0.6), 3(2.4,1.8) \\
5(4,3), 10(8,6) \\
20(16,12), 30(24,18)\end{array}$ & $6(3.6,1.2)$ & $4.5(2.73,9)$ & $78(103,151)$ & $2.4(2.4,1.7)$ & $.0768(.093, .143)$ \\
\hline 13 & .02 & $.08(.07, .06)$ & $.18(.15, .13)$ & $1.82(1.55,1.27)$ & 5.9 & $7(5.6,4.2)$ & $2.4(1.4,5)$ & $1.82(1.1, .4)$ & $78(103,151)$ & $2.4(2.43,1.69)$ & $.068(.093, .143)$ \\
\hline 14-Left & .029 & $.08(.06)$ & $.06(.04)$ & $.65(.45)$ & 5.9 & $5(4,3)$ & $6(3.6,1.2)$ & $4.5(2.7, .9)$ & $78(151)$ & $2.4(1.69)$ & $.068(.143)$ \\
\hline 14-Right & .173 & $.48(.35)$ & $.08(.05)$ & $.80(.55)$ & 5.9 & $5(4,3)$ & $6(3.6,1.2)$ & $4.5(2.7, .9)$ & $78(151)$ & $2.4(1.69)$ & $.068(.143)$ \\
\hline $15(\mathrm{SL})$ & .02 & $.08(.06)$ & $.18(.13)$ & $1.82(1.27)$ & 5.9 & $7(4.2)$ & $6(1.2)$ & $4.5(.9)$ & $78(c)$ & $2.4(c)$ & $.068(c)$ \\
\hline $15(3 \mathrm{~L})$ & - & $.10(.07)$ & - & $2(1.4)$ & 5.9 & $7(4.2)$ & - & $4.5(.9)$ & $78(c)$ & $2.4(c)$ & $.068(c)$ \\
\hline
\end{tabular}

Table 4: Intermediate and output model parameters (aged samples are represented within parentesis). The amplitude of the dermoepidermal junction is $A_{x, y}=100 \mu \mathrm{m}$ for all the 30-year old samples, and $A_{x, y}=0 \mu \mathrm{m}$ for 80-year old ones. Some values are the same for all images and not reported in the table. These are: Eumelanin concentration in melanosomes is $80 \mathrm{~g} / \mathrm{L}$. Pheomelanin concentration in melanosomes $12 \mathrm{~g} / \mathrm{L}$. Hb (oxy+deoxyb) in blood is $150 \mathrm{~g} / \mathrm{L} . \vartheta_{\mathrm{Hb}}$ is always set to $5 \%$ in HD. Bilirubin in blood is 0.05 $\mathrm{g} / \mathrm{L}$. $\beta$-carotene in blood is $7.0 e^{-5} \mathrm{~g} / \mathrm{L}$; in LE and $S C$, it is $2.1 e^{-4} \mathrm{~g} / \mathrm{L}$. The oxy-deoxy ratio of hemoglobin is $75 \%$. Water content, given in gram water per gram wet skin, is 5, 20,50,70, and 70 for $S C, L E, P D, R D$ and $H D$ respectively. Values labelled as c are kept constant for the purpose of isolating the influence of the studied parameter.

[BK10] BARANOSKI G. V., KRISHNaSwamy A.: Light and Skin Interactions: Simulations for Computer Graphics Applications. Morgan Kaufmann, 2010. 2

[BKTK00] BoIsSieuX L., Kiss G., Thalmann N. M., KALRA P.: Simulation of skin aging and wrinkles with cosmetics insight. In Computer Animation and Simulation 2000. Springer, 2000. 2

[CYL*02] Chung J. H., Yano K., Lee M. K., Youn C. S., Seo J. Y., Kim K. H., Cho K. H., Eun H. C., Detmar M.: Differential effects of photoaging vs intrinsic aging on the vascularization of human skin. Archives of dermatology 138, 11 (2002). 3, 6

[DHT*00] DebeVec P., Hawkins T., Tchou C., Duiker H.-P., SAROKIN W., SAGAR M.: Acquiring the reflectance field of a human face. In Proceedings of the 27th Annual Conference on Computer Graphics and Interactive Techniques (New York, NY, USA, 2000), SIGGRAPH '00, ACM Press/Addison-Wesley Publishing Co., pp. $145-156.2$

[dI11] D'EON E., IRving G.: A quantized-diffusion model for rendering translucent materials. ACM Transactions on Graphics 30, 4 (2011). 2, 7

[DJ05] DONNER C., JENSEN H. W.: Light diffusion in multi-layered translucent materials. ACM Transactions on Graphics 24, 3 (2005). $2,3,7$

[DJ06] DONNER C., JENSEN H. W.: A spectral bssrdf for shading human skin. In $E G S R$ '06 (2006). 2, 3, 6

[dL07] D'EON E., LuEBKE D.: Advanced techniques for realistic realtime skin rendering. In GPU Gems 3. Addison Wesley, 2007, ch. 14.7

[dLE07] D'EON E., LUEBKE D., ENDERTON E.: Efficient rendering of human skin. EGSR '07 (2007). 2, 7

[DWd*08] DONNER C., Weyrich T., D’EON E., RAMAMOORTHI R., RUSINKIEWICZ S.: A layered, heterogeneous reflectance model for acquiring and rendering human skin. ACM Transactions on Graphics 27, 5 (2008). 2, 8, 9

[EHM03] EdWARDS C., HEGGIE R., MARKs R.: A study of differences in surface roughness between sun-exposed and unexposed skin with age. Photodermatology, photoimmunology \& photomedicine 19, 4 (2003). 4
[EHT07] EgaWa M., HIRAO T., TAKAHASHI M.: In vivo estimation of stratum corneum thickness from water concentration profiles obtained with raman spectroscopy. Acta dermato-venereologica 87 , 1 (2007). 8

[Fit88] FitZPATRICK T. B.: The validity and practicality of sun-reactive skin types i through vi. Archives of dermatology 124, 6 (1988). 8

[FL86] FENSKE N. A., LOBER C. W.: Structural and functional changes of normal aging skin. Journal of the American Academy of Dermatology 15, 4 (1986), 571-585. 3

[FMM10a] Farage M. A., Miller K. W., Maibach H. I.: Degenerative changes in aging skin. In Textbook of aging skin. Springer, 2010. 1, 6

[FMM*10b] Farage M. A., Miller K. W., Maibach H. I., Farage M. A., Miller K. W., Maibach H. I.: Textbook of aging skin. Springer, 2010. 2

[GFT*11] Ghosh A., FyfFe G., Tunwattanapong B., Busch J., YU X., DeBEVEC P.: Multiview face capture using polarized spherical gradient illumination. ACM Transactions on Graphics 30, 6 (2011). 2

[GGF*10] Giangreco A., Goldie S. J., Failla V., Saintigny G., WATt F. M.: Human skin aging is associated with reduced expression of the stem cell markers $\beta 1$ integrin and mcsp. Journal of Investigative Dermatology 130, 2 (2010). 4

[GHP*08] Ghosh A., Hawkins T., Peers P., Frederiksen S. DEBEVEC P.: Practical modeling and acquisition of layered facial reflectance. ACM Transactions on Graphics 27, 5 (2008). 2

[GMP*06] Golovinskiy A., Matusik W., Pfister H., RUSinkIEwiCZ S., FUnKHOUSER T.: A statistical model for synthesis of detailed facial geometry. ACM Transactions on Graphics 25, 3 (2006). 2

[GS79] Gilchrest B. A., SZABo G.: Effects of aging and chronic sun exposure on melanocytes in human skin. Journal of Investigative Dermatology 73, 2 (1979). 6

[GTB*13] GRaham P., Tunwattanapong B., Busch J., YU X., JoNES A., DebeVEC P., GHosh A.: Measurement-based synthesis 
of facial microgeometry. Computer Graphics Forum 32, 2pt3 (2013). 2

[HCJ13] Habel R., Christensen P. H., Jarosz W.: Photon beam diffusion: A hybrid monte carlo method for subsurface scattering. Computer Graphics Forum 32, 4 (2013). 2

[INN07] IGARASHI T., Nishino K., NAYAR S. K.: The appearance of human skin: A survey. Foundations and Trends in Computer Graphics and Vision 3, 1 (2007). 2

[Jac13] JACQUES S. L.: Optical properties of biological tissues: a review. Physics in medicine and biology 58, 11 (2013). 5, 6, 7

[JB02] JENSEN H. W., BUHLER J.: A rapid hierarchical rendering technique for translucent materials. ACM Transactions on Graphics (TOG) 21, 3 (2002). 2, 7

[JMLH01] Jensen H. W., Marschner S. R., LeVoy M., HANRAHAN P.: A practical model for subsurface light transport. In Proceedings of the 28th Annual Conference on Computer Graphics and Interactive Techniques (New York, NY, USA, 2001), SIGGRAPH '01, ACM, pp. 511-518. 2

[JSB*10] Jimenez J., Scully T., Barbosa N., Donner C., Alvarez X., Vieira T., Matts P., Orvalho V., Gutierrez D., WEYRICH T.: A practical appearance model for dynamic facial color. ACM Transactions on Graphics 29, 6 (2010). 2, 8, 9

[JSG09] JimenEZ J., SundSTEDT V., GuTIERREZ D.: Screen-space perceptual rendering of human skin. ACM Transactions on Applied Perception 6, 4 (2009). 2, 7

[JWSG10] JimeneZ J., WhelAn D., SundSTEDT V., GutierREZ D.: Real-time realistic skin translucency. IEEE Computer Graphics and Applications 30, 4 (2010). 2

[JZJ*15] Jimenez J., Zsolnai K., Jarabo A., Freude C., AuZINGer T., WU X.-C., VON DER PAHLEN J., WiMmeR M., Gutierrez D.: Separable subsurface scattering. Computer Graphics Forum (2015). 2

[KB04] KRISHNASWAMY A., BARANOSKI G. V.: A biophysicallybased spectral model of light interaction with human skin. Computer Graphics Forum 23, 3 (2004). 2, 3, 4, 5

[LATO*97] LOCK-ANDERSEN J., THERKILDSEN P., OLIVARIUS F., Gniadecka M., Dahlstrem K., Poulsen T., Wulf H.-C.: Epidermal thickness, skin pigmentation and constitutive photosensitivity. Photodermatology, photoimmunology \& photomedicine 13, 4 (1997). 4

[MCD97] MATCHER S., COPE M., DELPY D.: In vivo measurements of the wavelength dependence of tissue-scattering coefficients between 760 and $900 \mathrm{~nm}$ measured with time-resolved spectroscopy. Applied Optics 36, 1 (1997). 7

[MFB*97] MOURANT J. R., Fuselier T., BOYER J., JOHNSON T. M., Bigio I. J.: Predictions and measurements of scattering and absorption over broad wavelength ranges in tissue phantoms. Applied Optics 36, 4 (1997). 7

[MHP*07] Ma W.-C., Hawkins T., PeErs P., Chabert C.-F., WeISS M., DEBEVEC P.: Rapid acquisition of specular and diffuse normal maps from polarized spherical gradient illumination. In EGSR '07 (2007). 2

[MM02] Meglinski I. V., MATCHER S. J.: Quantitative assessment of skin layers absorption and skin reflectance spectra simulation in the visible and near-infrared spectral regions. Physiological measurement 23, 4 (2002). 4, 5

[MWL*99] MARSChNeR S. R., Westin S. H., LAFORTUNE E. P., Torrance K. E., GreEnberG D. P.: Image-based BRDF measurement including human skin. In Rendering Techniques 1999. Springer, 1999. 2

(c) 2015 The Author(s)

Computer Graphics Forum (c) 2015 The Eurographics Association and John Wiley \& Sons Ltd.
[NBH*98] Nordlund J. J., Boissy R. E., HeARING V. J., KInG R. A., ORTONNE J.: The pigmentary system. Oxford University Press New York NY, 1998. 1, 2

[Pen62] PENndorf R.: Angular Mie scattering. JOSA 52, 4 (1962). 6 [PF97] POPE R. M., FRY E. S.: Absorption spectrum (380-700 nm) of pure water. ii. integrating cavity measurements. Applied optics 36, 33 (1997). 5

[PH10] PhARr M., Humphreys G.: Physically Based Rendering, Second Edition: From Theory To Implementation, 2nd ed. Morgan Kaufmann Publishers Inc., 2010. 7

[PSD79] Pochi P. E., Strauss J. S., Downing D. T.: Age-related changes in sebaceous gland activity. Journal of Investigative Dermatology 73, 1 (1979). 2, 4, 5

[Sai92] SAIDI I. S.: Transcutaneous optical measurement of hyperbilirubinemia in neonates. $\mathrm{PhD}$ thesis, Rice, University, 1992. http://hdl.handle.net/1911/19082. 5

[SJNY06] Salomatina E., JiAng B., NovaK J., YAROSLAVSKY A. N.: Optical properties of normal and cancerous human skin in the visible and near-infrared spectral range. Journal of Biomedical Optics 11, 6 (2006). 7

[Sta01] STAM J.: An illumination model for a skin layer bounded by rough surfaces. In Rendering Techniques 2001. Springer, 2001. 2

[THM99] Tsumura N., HANEISHI H., MiYAKE Y.: Independentcomponent analysis of skin color image. JOSA A 16, 9 (1999). 2

[TOS*03] Tsumura N., OJima N., Sato K., Shiraishi M., Shimizu H., Nabeshima H., AKaZAKI S., HoRi K., MiYaKe Y.: Image-based skin color and texture analysis/synthesis by extracting hemoglobin and melanin information in the skin. ACM Transactions on Graphics 22, 3 (2003). 2

[TS67] TORRANCE K. E., SPARROW E. M.: Theory for off-specular reflection from roughened surfaces. JOSA 57, 9 (1967). 4

[Tuc07] TUCHIN V. V.: Tissue optics: light scattering methods and instruments for medical diagnosis, vol. 13. SPIE press Bellingham, 2007. 2

[WE73] Whitton J. T., Everall J.: The thickness of the epidermis. British Journal of dermatology 89, 5 (1973). 8

[WJZ95] WANG L., JACQUES S. L., ZHENG L.: Mcml - monte carlo modeling of light transport in multi-layered tissues. Computer methods and programs in biomedicine 47, 2 (1995). 7

[WM05] WALlER J. M., MAIBACH H. I.: Age and skin structure and function, a quantitative approach (i): blood flow, ph, thickness, and ultrasound echogenicity. Skin Research and Technology 11, 4 (2005). 4

[WMP*06] Weyrich T., Matusik W., Pfister H., Bickel B., Donner C., Tu C., McAndless J., Lee J., NGan A., Jensen H. W., ET AL.: Analysis of human faces using a measurement-based skin reflectance model. ACM Transactions on Graphics 25, 3 (2006). 2

[YMOI12] YoshidA K., MiYAKI M., OJIMA N., IwATA K.: Relationship between microstructure of the skin surface and surface reflection based on geometric optics. Journal of Dermatological Science 66, 3 (2012). 2, 4

[ZD09] Zonios G., Dimou A.: Light scattering spectroscopy of human skin in vivo. Optics Express 17, 3 (2009). 7 